\title{
Minijobs in der Gebäudereinigung
}

\author{
PETER RIEDEL
}

Die Problematik der Minijobs in der Gebäudereinigung kann hier nur in einigen Aspekten angerissen werden. Vorrangig geht es darum, zunächst die Bedeutung der Minijobs in dieser Branche anhand von Daten und Fakten kurz darzustellen und die tarifrechtliche wie auch gesetzliche Regulierung dieser Beschäftigungsform im Bereich der IG Bauen-Agrar-Umwelt (BAU) zu umreißen. Die immer wiederkehrenden Versuche, die individuellen wie auch kollektiven Rechte von Minijobberinnen und Minijobbern zu unterlaufen („dirty tricks“), werden mit konkreten Praxisbeispielen illustriert. Abschließend werden knappe Überlegungen angestellt, welche Konsequenzen daraus für die gewerkschaftliche Positionierung zum Thema „Minijobs“ resultieren können.

Die Datenlage zu den oben genannten Aspekten ist in großen Teilen ungenügend, sodass der Beitrag sich auch auf die Erfahrungen und Recherchen der IG BAU und ihrer Beschäftigten wie auch auf Gespräche mit Beschäftigten aus der Branche stützt. ${ }^{\mathbf{0}}$ Vor allem wurde auch auf die Kompetenzen der Abteilung Frauen und der sozialpolitischen Abteilung der IG BAU zurückgegriffen, die das Thema unter ihrem spezifischen Blickwinkel bearbeiten. ${ }^{2}$

Die Begriffe Minijob und geringfügiges Beschäftigungsverhältnis werden im Rahmen dieser Ausführungen synonym gebraucht.

\section{Daten und Fakten}

Die Gebäudereinigung ist mit 873.723 Erwerbstätigen in 34.469 Betrieben das beschäftigungsstärkste Handwerk in Deutschland. ${ }^{3}$ Davon arbeiten 457.420 Personen in einem geringfügigen Beschäftigungsverhältnis. ${ }^{\mathbb{0}}$ Die Zahl der geringfügigen Beschäftigungsverhältnisse ist im Zeitraum von 1999 bis 2009 um 55,4\% gewachsen, wohingegen die Anzahl der Gesamtbeschäftigten im nahezu vergleichbarem Zeitraum lediglich um $15 \%$ gestiegen ist. Gut ein Drittel der geringfügig Beschäftigten übt den Minijob zusätzlich zu einer sozialversicherungspflichtigen Beschäftigung aus.

Die Gebäudereinigungsbranche ist dadurch geprägt, dass mehr als die Hälfte der Beschäftigten nur ein geringfügiges Arbeitsverhältnis hat. Die Unternehmen scheinen dieses Verhältnis als Strategie und Notwendigkeit zu betrachten, um den Kunden entsprechende Flexibilität in der Erbringung von Reinigungsleistungen anbieten zu können. ${ }^{\ominus}$ Die IG BAU als tarifpolitisch zuständige Gewerkschaft für den Reinigungssektor in Deutschland vertritt dagegen die Auffassung, dass eine Zunahme an Minijobs eher zur Ver- drängung regulärer Beschäftigungsverhältnisse führt als zusätzliche Arbeitsplätze für Arbeitslose zu schaffen. Darüber hinaus stellen die Gewerkschaftssekretäre und -sekretärinnen fest, dass Minijobbern oftmals die zustehenden tariflichen oder arbeitsrechtlichen Ansprüche nicht gewährt werden. ${ }^{\circ}$

\section{Tarifvertragliche und gesetzliche Regulierung}

Die zwischen der IG BAU und dem Bundesinnungsverband des Gebäudereiniger-Handwerks (BIV) abgeschlossenen Tarifverträge gelten für Minijobber mit gleichen Rechten und Pflichten wie für Teil- oder Vollzeitbeschäftigte. Der Rahmentarifvertrag für die gewerblich Beschäftigten in der Gebäudereinigung ist allgemeinverbindlich erklärt und gilt zwingend, unabhängig davon, ob Unternehmen Mitglied des Arbeitgeberverbandes oder Beschäftigte Mitglied der IG BAU sind. Dasselbe gilt für den Mindestlohntarifvertrag in der Gebäudereinigung. Das Entsendegesetz und die Finanzkontrolle Schwarzarbeit erzeugen mit den damit verbundenen Sanktionen bei Verstößen eine noch höhere Verbindlichkeit der Einhaltung.

Seit 2007 gibt es in Ost- und Westdeutschland in der Gebäudereinigung allgemeinverbindlich erklärte Mindest(stunden)löhne für die Innen- und Unterhaltsreinigung (Lohngruppe 1) und für die Glas- und Fassadenreinigung

(1) Aktuell hat der Vorstandsbereich VII - Dienstleistungen der IG Bauen-Agrar-Umwelt eine Umfrage unter den Beschäftigten der Gebäudereinigung zu dieser Thematik gestartet, um einen größeren Datenfundus zu erhalten. Erste Ergebnisse sind aber nicht vor der Jahreswende 2011/12 zu erwarten.

2 Vgl. IG Bauen-Agrar-Umwelt, Vorstandsbereich IV - Frauen (Hrsg.) (2011): Fraueninfo (29), Frankfurt a. M.

(3) Quelle: http://www.gebaeudereiniger.de/uploads/media/ Anlage_Daten_und_Fakten_20100526.pdf.

(4) Stand Dezember 2009. Quelle: Statistik der Bundesagentur für Arbeit; Berechnungen des Autors.

5 Vgl. Grömling, M. (2006): Branchenreport GebäudereinigerHandwerk, hrsg. vom Institut der deutschen Wirtschaft (IW), Köln.

(6 Vgl. Bundesvorstand der IG Bauen-Agrar-Umwelt (Hrsg.) (2011): Gute Arbeitsplätze statt billiger Minijobs, Diskussionspapier, Frankfurt a. M. 
(Lohngruppe 6). Diese Stundenlöhne gelten zwingend für alle Beschäftigten, die Tätigkeiten in der Gebäudereinigung ausüben.

Die Tarifvertragsparteien haben „anlässlich des fünften Bündnisgespräches gegen Schwarzarbeit im Gebäudereiniger-Handwerk am 28. Januar 2011 in Bonn festgestellt, dass rund $90 \%$ der geprüften Betriebe im GebäudereinigerHandwerk den allgemeinverbindlichen Mindestlohn zahlen. Dies belegen die Ergebnisse der letzten Prüfungen der Fi-

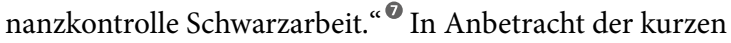
Zeitspanne des Wirkens des Mindestlohntarifvertrages ist das ein sehr positives Ergebnis. Dennoch ist vor dem Hintergrund der großen Zahl an Reinigungsunternehmen in Deutschland, der wachsenden Anzahl an Mindestlohnbranchen und der begrenzten Anzahl der Mitarbeiter der Finanzkontrolle der Anteil der „schwarzen Schafe“ in dieser Branche nicht zu unterschätzen.

Des Weiteren gelten die Tarifverträge zur Altersvorsorge, zum zusätzlichen Urlaubsgeld sowie der Lohntarifvertrag in der Gebäudereinigung natürlich auch für Minijobber, vorausgesetzt es liegt eine beidseitige Tarifbindung vor. Gleichwohl gibt es in der Praxis, im Arbeitsalltag der Minijobbeschäftigten, immer wieder Versuche, die rechtlichen Standards zu unterlaufen.

\section{„Dirty Tricks“ im Umgang mit Minijobbern}

Ein „dirty trick“ von Unternehmen ist, den tariflichen bzw. allgemeinverbindlichen Stundenlohn in der Gebäudereinigung ,illegal“ zu akkordieren. Dies wird durch unterschiedliche Methoden erreicht: Den Reinigungsbeschäftigten wird entweder ihre Arbeitszeit bei gleichbleibender Reinigungsleistung gekürzt, oder die Reinigungsleistung wird bei gleichbleibender Arbeitszeit erhöht (beispielsweise durch Erweitern der Reinigungsfläche). ${ }^{\circledR}$

Die Reinigungsbeschäftigten erhalten dasselbe Einkommen bei gestiegener Arbeitsbelastung, obwohl der Stundenlohn auf der Abrechnung ,augenscheinlich“ höher ist. Dabei müsste aber jede Tariferhöhung bei geringfügig Beschäftigten an der 400-€-Grenze zu Arbeitszeitverkürzung bei vollem Lohnausgleich führen. Das Ergebnis dieser Methoden ist für die Beschäftigten fatal, weil sich die Arbeitsintensität immer weiter erhöht. Für Unternehmen sind sie aber wirtschaftlich anscheinend so lukrativ, dass sie lieber die $10 \%$ höhere Pauschalabgabe für einen Minijob bezahlen, als ein reguläres Arbeitsverhältnis in Teilzeit oder Vollzeit anzubieten.

Die IG BAU hat deshalb gemeinsam mit der RAL Gütegemeinschaft Gebäudereinigung e.V. eine bundesweite Kampagne unter dem Motto „Sauberkeit braucht ihre Zeit“ gestartet. Ziel dieser Initiative ist es, Kunden bei Ausschreibung von Reinigungsleistungen, Reinigungskräfte und Unternehmen der Gebäudereinigung zu sensibilisieren und den explosionsartigen Anstieg der zu reinigenden Flächen durch Vorgabe von definierten Zeitwerten zu begrenzen. ${ }^{\circledR}$

Aktuell werden zunehmend auch wieder sogenannte Objektlöhne eingeführt. Unternehmen vereinbaren mit den
Reinigungsbeschäftigten arbeitsvertraglich im Rahmen der 400-€-Grenze eine Arbeitszeit für eine festgelegte Objektpauschale. Üblicherweise ist dann die Reinigungsleistung innerhalb der vereinbarten Zeit nicht zu schaffen, unbezahlte Mehrarbeit wird quasi einkalkuliert und häufig durch die Beschäftigten auch geleistet. Dennoch wird nur der vereinbarte Objektlohn abgerechnet. Arbeitszeitnachweise nach den Verordnungen des Entsendegesetzes würden zwar diese illegale Verfahrensweise entlarven, werden aber selten von den Beschäftigten geführt und liegen deshalb als notwendiger Beweis eines Mindestlohnverstoßes für das Kontrollorgan, die Finanzkontrolle Schwarzarbeit, nicht oder häufig nur unzulänglich vor.

Das Vorenthalten von Urlaubsansprüchen, Wegezeitleistungen, der Lohnfortzahlung im Krankheitsfall oder Erschwerniszuschlägen sind weitere Missstände, die die IG BAU bei der Beschäftigung von Minijobbern immer wieder feststellt.

Gerade das Problem der Vergütung von Wegezeiten hat für die geringfügig Beschäftigten einen zunehmend höheren Stellenwert. Minijobberinnen und Minijobber werden von Unternehmen sehr flexibel eingesetzt. Sie haben oftmals mehrere Objekte am Tag zu reinigen. Den Reinigungskräften wird dann der Anspruch auf Wegezeitenvergütung vorenthalten, häufig mit dem Argument, dass sie sich im Arbeitsvertrag zu dem sogenannten "geteilten Dienst“ verpflichtet hätten. Dieser ,geteilte Dienst“ ist ein umstrittener Rechtsbegriff, der in den Tarifverträgen der Gebäudereinigung keine Grundlage hat. Zwar haben die IG BAU und der BIV diesbezüglich rechtsverbindliche Regelungen geschaffen, die aber in ihrer Auslegung zwischen den Tarifvertragsparteien strittig sind und in der Praxis kaum zur Anwendung kommen. ${ }^{\oplus}$ Mittlerweile haben die Tarifvertragsparteien durch die redaktionelle Novellierung des Rahmentarifvertrags (RTV) in der Gebäudereinigung diese Problematik neu geregelt, der RTV ist aber aktuell noch nicht in Kraft und wird sich dann in der Praxis auch noch beweisen müssen.

Eine gesonderte Stellung nimmt das befristete Arbeitsverhältnis von Minijobbern ein. In der Gebäudereinigung ist es mittlerweile zur Normalität geworden, dass bei

$(7$ Quelle: http://www.gebaeudereiniger.de/1592.html.

(8 Sehr anschaulich wird das Problem der Flächenleistungen in den Filmen „Die Probe aufs Exempel - Leistungszahlen im Praxistest" (http://www.youtube.com/ watch? $v=$ WDNIRci48Tw) und "Büroreinigung in 4 Minuten. Unfassbar!“ (http://www.youtube.com/watch?v=Ch4TTUx5dA und http://www.youtube.com/watch?v=RGJWx_zj2As) dargestellt.

(9 Vgl. IG Bauen-Agrar-Umwelt. Vorstandsbereich VII - Dienstleistungen (Hrsg.) (2010): Sauberkeit braucht ihre Zeit, Frankfurt a. M.

(1) Vgl. Rahmentarifvertrag für die gewerblichen Beschäftigten in der Gebäudereinigung vom 4. Oktober 2003. 
der Neueinstellung von Beschäftigten nicht nur eine Probezeit vereinbart, sondern dass das Arbeitsverhältnis auch gleichzeitig befristet wird. ${ }^{\circledR}$ Das berühmte „Damoklesschwert“ hängt dann über dem „Kopf“ der befristet Beschäftigten. Damit steigt der physische wie psychische Druck, nicht „negativ“ gegenüber dem Unternehmen aufzufallen, um eine eventuelle Weiterbeschäftigung zu erhalten. ${ }^{\text {(3) }}$ Gegenüber der betrieblichen Interessenvertretung oder den Gewerkschaften verhalten sich befristet Beschäftigte oft wie gelähmt, sie wagen es nicht, für ihre Rechte einzustehen.

Zusammenfassend ist festzuhalten, dass Minijobberinnen und Minijobber häufig faktisch als billige Arbeitnehmer „Zweiter Klasse“ behandelt werden, obwohl sie theoretisch Anspruch auf eine Vielzahl tarifvertraglicher Leistungen haben.

\section{Geringfügig Beschäftigte in der IG BAU}

Der Anteil von geringfügig Beschäftigten spielt im Mitgliederbestand der Gebäudereinigung bei der IG BAU eine nicht unerhebliche Rolle. Zwar hat der Anteil von geringfügig Beschäftigten von Dezember 2005 bis Dezember 2010 von 25,3 auf 20,8\% abgenommen, dennoch sind 12.212 Mitglieder in Minijobs zum Gesamtmitgliederbestand von 58.691 eine gewichtige politische Größe in der Gebäudereinigung der IG BAU (Stand: 12/2010). Darüber hinaus besetzen geringfügig Beschäftigte mittlerweile auch einige Mandate in den entscheidenden Satzungsgremien der Organisation und können somit direkten Einfluss auf die politische Ausrichtung der Gesamtorganisation nehmen.

Dass sich Reinigungskräfte mehr und mehr politisch engagieren und den Respekt für ihre Arbeit einfordern, zeigt auch das kontinuierliche Mitgliederwachstum bei der IG BAU im Reinigungssektor. In den letzten fünf Jahren hat die IG BAU in der Gebäudereinigung einen Mitgliederzuwachs von rund $25 \%$ zu verzeichnen, der mit wenigen Schwankungen bislang auch im Jahr 2011 anhält. ${ }^{\text {. }}$ Eine ähnliche Entwicklung lässt sich bei den monatlichen Neuaufnahmen in der Gebäudereinigung seit 2006 feststellen. Rund $20 \%$ der monatlichen Neuaufnahmen in der Gebäudereinigung sind geringfügig Beschäftigte. ${ }^{(0)}$

\section{Minijobber und Streikrecht}

Im ersten bundesweiten Arbeitskampf in der Gebäudereinigung in den Monaten September und Oktober 2009 haben sich entsprechend den Anteilen an der Gesamtorganisation die geringfügig Beschäftigten aktiv beteiligt. 15.340 Streikende konnten einschließlich der Warnstreiks an allen Streiktagen verzeichnet werden. Es ist schwer einzuschätzen und zu belegen, ob die Motivation zur Beteiligung eher durch ein ,ist mir egal, ich habe nichts zu verlieren“ bewirkt wurde, oder ob der Wille, für deutlich mehr Geld zu streiken, ausschlaggebend war, und zwar trotz der weit verbreiteten Angst, den Job verlieren zu können. Sicherlich sind die Gründe bzw. die Motivation zu streiken noch viel differenzierter zu betrachten, was an dieser Stelle jedoch zu weit führen würde.

Die Arbeitgeber hatten Objekte mit vielen geringfügig Beschäftigten als strategische Schwachpunkte eingeschätzt; das war im Streik offensichtlich zu erkennen. Gerade in Reinigungsobjekten mit mehrheitlich geringfügig Beschäftigten wurde versucht, über Streikbrecher (in der Regel Subunternehmer - oftmals auch mit hohen Anteilen geringfügig Beschäftigter) die ausgefallene Arbeit nachzuholen. Nicht selten ging die Strategie der Arbeitgeber ins Leere. Permanentes Rollieren in den Streikobjekten führte zu Verwirrung im Einsatz von Streikbrechern, die dann vor einem vermeintlichem Streikobjekt standen, in dem alle vormals streikenden Reinigungskräfte wieder arbeiteten. Das Unternehmen erlitt wirtschaftlichen Schaden durch zusätzliche Zahlung des Subunternehmers, ohne dass dieser eine Arbeitsleistung erbringen konnte.

Von subtilem Druck auf die Familien der Streikenden und auf die Streikenden selbst wurde zwar in Einzelfällen berichtet, aber weder Abmahnungs- oder Kündigungswellen noch andere Repressalien waren während und nach dem Streik überproportional festzustellen. Wie die Unternehmen mit streikenden Minijobbern in befristeten Arbeitsverhältnissen umgegangen sind, lässt sich nicht eindeutig bestimmen. Gespräche mit Betriebsräten in der Gebäudereinigung lassen zumindest den Verdacht aufkeimen, dass eine Reihe befristeter Arbeitsverhältnisse nach dem Arbeitskampf nicht fortgeführt bzw. nicht verlängert wurde.

\section{Fazit}

Der tarifvertragliche Schutz der geringfügig Beschäftigten ist in Deutschland flächendeckend gegeben. Er ist aber entscheidend davon abhängig, inwieweit die Beschäftigten diese ihnen zustehenden Rechte kennen und einfordern. Kontinuierliche Information über die Rechte als Minijobber durch

(11) Konkrete statistische Daten zu befristeten Arbeitsverhältnissen von Minijobbern in der Gebäudereinigung liegen nicht vor, aber eine Analyse des Instituts für Arbeitsmarktund Berufsforschung ist zu dem Ergebnis gekommen, dass "fast jeder zweite Arbeitnehmer in Deutschland zunächst befristet eingestellt" wird: siehe Institut für Arbeitsmarktund Berufsforschung (Hrsg.) (2010): Betriebspanel. Anteil der befristeten Neueinstellungen an allen Neueinstellungen, 24. März.

(12) Vgl. Giesecke, J./Groß, M. (2006): Befristete Beschäftigung, in: WSI-Mitteilungen 56 (5), S. 247-254.

(13 Quelle: IG Bauen-Agrar-Umwelt: interne Statistik; Berechnungen des Autors.

(4) Quelle: IG Bauen-Agrar-Umwelt: interne Statistik; Berechnungen des Autors. 
die Tarifvertragsparteien ${ }^{\circledR}$ und den Gesetzgeber könnten eine kurzfristige Maßnahme sein, Defizite im Umgang mit Minijobs und Rechtsbrüche zu minimieren. Die Position von Minijobberinnen und Minijobbern sollte u.a. durch die folgenden politischen Maßnahmen gestärkt werden:

- eine verbesserte Informations- und Dokumentationspflicht des Arbeitgebers gegenüber den geringfügig Beschäftigten bezüglich ihrer Ansprüche auf bezahlten Urlaub, Lohnfortzahlung und ggf. Tariflohn bzw. Mindest-Stundenlohn;

- Vorrang für Minijobber, wenn ihr Arbeitgeber reguläre Stellen neu besetzt;

- Wiedereinführung einer Stundenobergrenze, deren Höhe sich u.a. aus allgemeinverbindlichen Mindestlöhnen ergibt;

- Änderung der „Hartz IV“-Regeln für eine „zumutbare“ Tätigkeit, sodass nur tariflich (bzw. ortsüblich) entlohnte und sozialversicherungspflichtige Tätigkeiten aufgenommen werden müssen.

Wenn immer mehr geringfügig Beschäftigte ihre Rechte einfordern, verlieren Minijobs für Arbeitgeber an Attraktivität. Langfristig ist das System mit Blick auf die Sozialversicherungssysteme, die Zunahme der Altersarmut und die

(15) Vgl. IG Bauen-Agrar-Umwelt, Vorstandsbereich IV - Frauen (Hrsg.) (2010): Meine Rechte im Minijob, Frankfurt a. M.
Sicherung von zukunftsfähigen Arbeitsplätzen sowie dem hohen Anteil der fast schon „kriminellen“Verfahrensweisen im Umgang mit geringfügigen Beschäftigungsverhältnissen grundlegend zu überdenken. Ziel ist dabei die grundsätzliche Einbeziehung aller Arbeitsverhältnisse in die gesetzlichen Sozialversicherungen und die Gleichbehandlung aller Arbeitsverhältnisse. Die Herausforderung bleibt, gemeinsam mit geringfügig Beschäftigten konkrete Gestaltungsvorschläge und überzeugende Wege zur Erreichung dieses Ziels zu entwickeln.

Befristungen der Arbeitsverhältnisse erschweren zusätzlich die Arbeit der betrieblichen sowie gewerkschaftlichen Interessenvertretung und verhindern die Gegenwehr der Betroffenen. Hier sind die Zielsetzungen des „Teilzeit- und Befristungsgesetzes“ genau zu prüfen und entsprechende gesetzliche Änderungen vorzunehmen.

\section{AUTOR}

PETER RIEDEL, M.A., IG Bauen-Agrar-Umwelt, Frankfurt a. M., Leiter der Abteilung Dienstleistungen im Vorstandsbereich VII - Dienstleistungen. Arbeitsschwerpunkte: Tarif-, Arbeits- und Sozialpolitik im Dienstleistungssektor, Branchen- Projekt- und Kampagnenarbeit im Dienstleistungssektor.

peter.riedel@igbau.de 